\title{
ENERGY AWARE TDMA MAC FOR WIRELESS SENSOR NETWORKS
}

\author{
S.Koteswararao ${ }^{1}$, Dr.M.Sailaja ${ }^{2}$, Dr.T.Madhu ${ }^{3}$, P.Ramesh ${ }^{4}$, V.Rajesh5 \\ 1,4,5 Department of Electronics and Communication Engineering, RIT, Yanam,U.T. \\ ${ }^{1}$ steevan2@gmail.com, ${ }^{4}$ pasupuleti.ramesh $87 @ g m a i l . c o m,{ }^{5}$ rit.hodece@yahoo.co \\ $\mathrm{m}$ \\ ${ }^{2}$ Department of Electronics and Communication \\ Engineering,JNTUK,Kakinada,A.P. \\ ${ }^{2}$ s.maruvada@gmail. com \\ ${ }^{3}$ Department of Electronics and Communication Engineering,SIET,Narsapur,A.P. \\ ${ }^{3}$ tennetimadhu@yahoo.com
}

\begin{abstract}
:
A novel approach for energy-aware management of sensor networks that maximizes the life of the sensors while maintaining desired quality of service attributes related to sensed data delivery is presented. This approach is to dynamically set routes and arbitrate medium access to minimize energy consumption and maximize sensor life. It presents a brief overview of the dynamic source routing and describes a TDMA based medium access control protocol (MAC). Hence the proposed APCRP-MAC Protocol improves the reliability and increases the life time of the sensor networks by reducing its power consumption. The new clustering and routing algorithm's scale well and converge fast for large scale dynamic sensors as shown by our extensive results using the Network Simulator (NS2).
\end{abstract}

Keywords: APCRP, TDMA, MAC, Energy Aware, NS-2.

\section{Introduction}

Wireless Sensor Networks (WSNs) are formed by hundreds or thousands of nodes that gather information and forward it to a sink node. Distinguished from traditional wireless networks, sensor networks are characterized of severe power computation, and memory constraints [1].Sensor networks have emerged as one of the dominant technology trends of this decade. Sensors and actuators, wireless communications and embedded computing are not new concepts but the recent low-cost large-scale integration of computation, communication and sensing into "wireless sensor networks" that has captured the attention of many researchers. Sensor networks enable observing the physical world at a granularity level which was unperceived before. Its infrastructure is composed of a large number of sensor nodes with a limited physical capacity and low cost. The energy consumption must be as optimized as possible in order to extend its lifetime. WSN for monitoring applications can benefit from this technique as it may maximize the lifetime of batteries. To validate the proposal simulation experiments have been performed using the Network Simulator (NS-2) tool. 


\subsection{Wireless sensor network}

Wireless sensor networking has potential usage in environment monitoring, defense, smart spaces, scientific application, medical systems, robotic exploration, target tracking, intrusion detection, wildlife habitat monitoring, climate control and disaster management etc. Energy efficiency is a critical issue in wireless sensor networks since batteries are the only energy source to power the sensor nodes [2]. In a sensor node, there are three activities which are main sources of energy consumption. Those activities are - (i) sensing, (ii) computation, and (iii) radio operations. Out of those three sources, energy loss due to radio operation is the maximum one.

\subsection{Sensor nodes:}

In addition to one or more sensors each node in a sensor network is typically equipped with a radio transceiver or other wireless communications device, a small microcontroller and an energy source usually a battery.

\subsection{Characteristics:}

The main characteristics of a WSN include: Power consumption constrains for nodes using batteries or energy harvesting ability to cope with node failures, Mobility of nodes, Dynamic network topology, Communication failures, Heterogeneity of nodes, Scalability to large scale of deployment, Ability to withstand harsh environmental conditions[3].

\subsection{Medium Access Control Layer:}

The Media Access Control (MAC) data communication protocol sub-layer, also known as the Medium Access Control is a sub layer of the Data Link Layer specified in the seven-layer OSI model (layer 2). It provides addressing and channel access control mechanisms that make it possible for several terminals or network nodes to communicate within a multi-point network typically a local area network (LAN) or metropolitan area network (MAN). The hardware that implements the MAC is referred to as a Medium Access Controller. The MAC sub-layer acts as an interface between the Logical Link Control (LLC) sub layer and the network's physical layer. The MAC layer emulates a full-duplex logical communication channel in a multi-point network [7]. This channel may provide uni-cast and multicast or broadcast communication service.

A communications protocol is a formal description of digital message formats and the rules for exchanging those messages in or between computing systems and in telecommunications. In MAC protocol the power control issues had taken an idea of sourcing the protocols in a wireless sensor networks. 


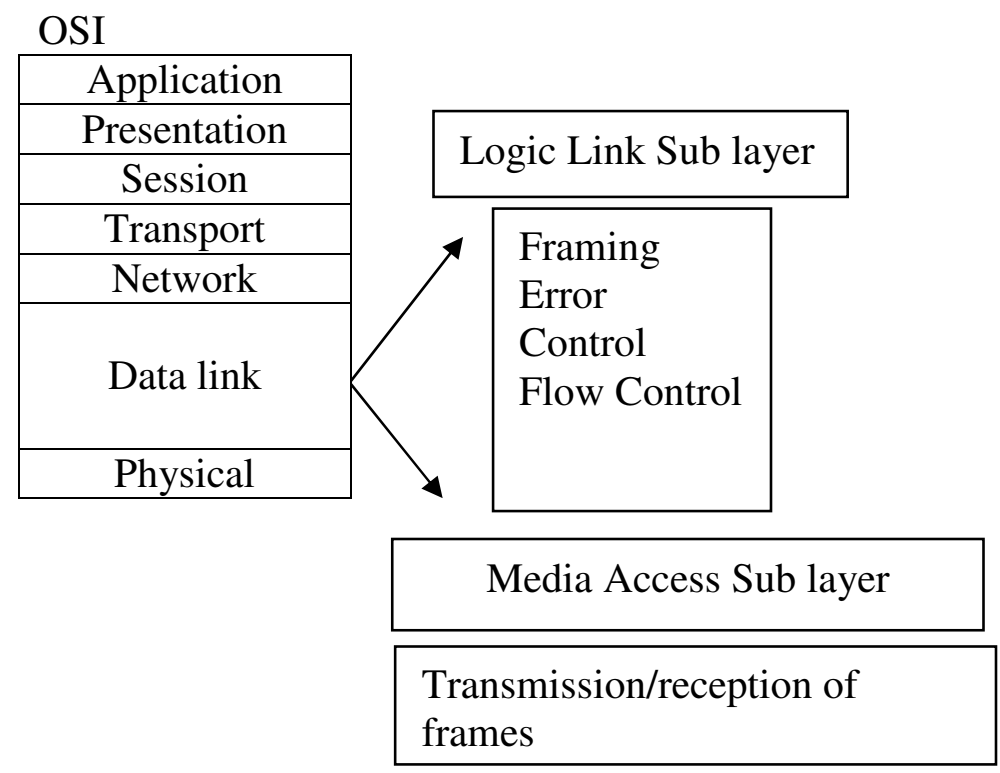

Figure 1.Layers of Mac Layer

\section{Causes of Energy Waste}

\subsection{Energy Advantage:}

Because of the unique attenuation characteristics of Radio frequency (RF) signals, a multi-hop RF network provides a significant energy saving over a single hop network for the same distance. Consider the following simple example of an N-hop network. Assume the overall distance for transmission distance is $\mathrm{Nr}$, where $r$ is the one-hop distance [4]. The minimum receiving power at a node for a given transmission error rate is $\mathrm{P}_{\text {receive, }}$, and the power at a transmission node is $\mathrm{P}_{\text {send }}$. Then the RF attenuation model near the ground is given by

$$
\mathrm{P}_{\text {receive }} \alpha \frac{P_{\text {send }}}{r^{\alpha}}
$$

Where $r$ is the transmission distance and $\alpha$ is the RF attenuation exponent. Due to multipath and other interference effects $\alpha$ is typically in the range of 2 to 5 equivalently,

$$
\mathrm{P}_{\text {send }} \alpha \mathrm{r}^{\alpha} \mathrm{P}_{\text {receive }} \text {. }
$$

Therefore, the power advantage of an N-hop transmission versus a single-hop transmission over the same distance $\mathrm{Nr}$ is

$$
\eta_{\mathrm{rf}}=\frac{P_{\text {send }(\mathrm{N})}}{N p_{\text {send }(r)}}=\frac{(N r)^{\alpha} p_{\text {receive }}}{N r^{\alpha} P_{\text {receive }}}=\mathrm{N}^{\alpha-1}
$$

A larger $\mathrm{N}$ gives a larger power saving due to the consideration of RF energy alone. However this analysis ignores the power usage by other components of an RF circuitry. Using more nodes increases not only the cost but also the power consumption of these other RF components. In practice, an optimal design seeks to balance the two conflicting factors for an 
overall cost and energy efficiency [6]. Latency and robustness considerations may also argue against an unduly large number of relay nodes.

The major causes of energy waste at MAC layer are collision, idle-listening, overhearing and control packet overhead [8]. Collision occurs when the transmission packets are corrupted partially or fully. Collision causes retransmission thus increases the latency and power consumption. Listening to an idle channel for possible traffic is called idle-listening. In order to conserve energy most of the energy efficient protocols put their network interface in sleep state rather than in idle state [9]. Receiving packets which are meant for other nodes is called over-hearing. Control packet overhead occurs when more number of control packets are used. Use of more numbers of control packets not only increase the energy consumption but also decrease the utilization of limited bandwidth. Carrier sensing is another major cause of energy waste due to collision of data packets and hidden and exposed terminal problems. Random access collision and the consequent retransmission: Collisions should be avoided as far as possible since otherwise the followed retransmission will lead to unnecessary energy consumption and longer time delay. Actually one of the fundamental tasks of any MAC protocol is to avoid collisions so that two interfering nodes do not transmit at the same time. The simplest ways for collision avoidance in a general network include code division multiple access (CDMA), time division multiple access (TDMA) and frequency division multiple access (FDMA). However, for mobile ad hoc networks there exist many special issues that need to be addressed for a MAC protocol design [5]. Idle listening: energy-aware MAC protocols that require nodes be in sleep mode periodically for energy conservation had been proposed. When in sleep mode, nodes neither transmit nor receive packets; but they must be woken up to idle mode first for attending trace relay. Sleep mode requires more than an order of magnitude less power than idle mode. Hence, intelligently switching to sleep mode whenever possible will generally lead to significant energy saving.

\subsection{Overhearing:}

Wireless nodes consume power unnecessarily due to overhearing the transmissions of their neighbors. This is often the case in a typical broad- cast environment. Control overhead, Protocol overhead should be reduced as much as possible, especially for transmitting short packets [10]. Due to the large channel acquisition overhead, small packets have disproportionately high energy costs. Header compression can be used to reduce packet length, thus achieving energy savings. Since significant energy is consumed by the mobile radio when switching between transmit and receive modes, packet aggregation for header overhead reduction will be useful. When mobile nodes request multiple transmission slots with a single reservation packet, the control overhead for reservation can be reduced [11]. Allocating contiguous slots for transmission or reception to reduce the turnaround also helps to achieve the low power consumption.

\section{TDMA (Time Division Multiple Access)}

In TDMA systems, the time axis is divided into the number of frames with fixed length. Each frame is further divided into a number of time slots. A user can only transmit in the pre-assigned time slots. By this way, interference from neighboring nodes is reduced. Compared with random access MAC protocols, TDMA protocols is advantageous in energy conservation. This is because of dedicated time slots for each user have been pre-determined. 
Therefore collision and the corresponding overhead can be avoided. However, the scalability of TDMA is not as good as that of random access scheme. Applying TDMA protocols in wireless systems usually require all nodes to form real communication clusters, such as in Bluetooth and LEACH. The control of inter cluster communication and interference is complex, especially when nodes are mobile as in MANETs. Further, TDMA requires the sender and receiver to be perfectly time-synchronized, which is not trivial. Designers need to consider factors such as the timing difference, clock shift, propagation delay etc [12].

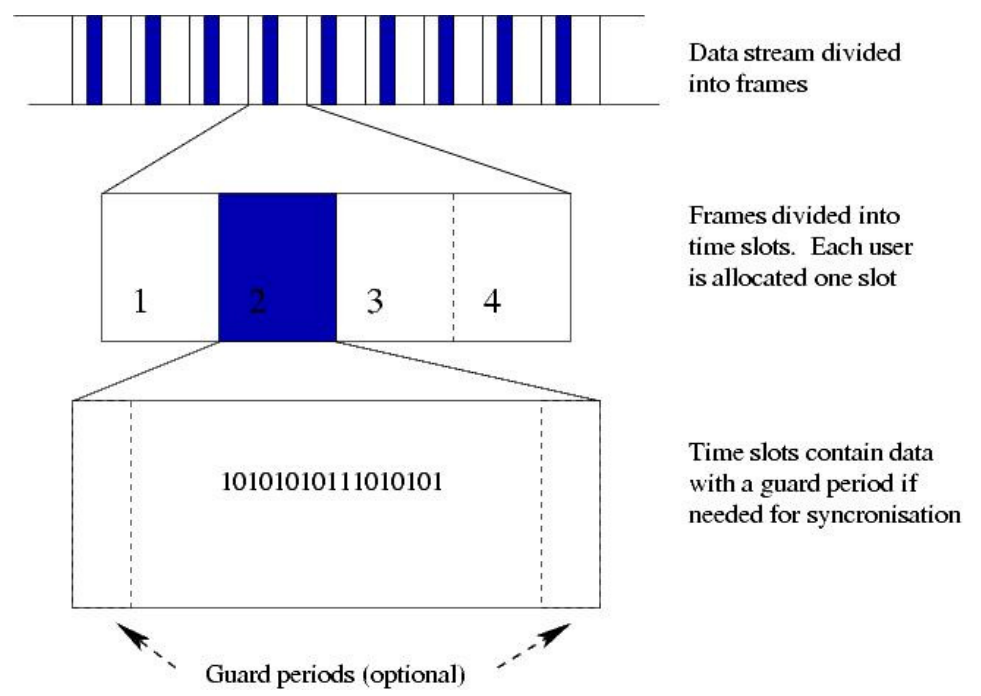

Figure 2.TDMA frame structure

\section{MAC Layer Protocol using TDMA}

Although the new routing protocol is independent of the MAC layer protocol choosing a certain MAC layer protocol may enhance the performance. In a wireless system the medium access protocols can be adapted and tuned to enhance energy efficiency. We choose to implement a time division multiple access (TDMA) based MAC layer whose slot assignment is managed by the gateway. The gateway informs each node about slots in which it should listen to other node's transmission and about the slots which the node can use for its own transmission [13].

The advantages of using a TDMA MAC layer are: Clock synchronization is built in the TDMA protocol. Recall that we need synchronization for the energy model refresh and sending rerouting decision from the gateway to the nodes.Collision among the nodes can be avoided since each node has its own assigned time slots [14]. Problems can occur with the existence of communication errors: a packet containing the slot assignment can be dropped. If a node that does not hear the gateway decision turns itself off, then no collision can occur.

\subsection{RTS/CTS (Request to Send/ Clear to Send):}

It uses RTS/CTS mechanisms and these signals are transmitted over the control channel while the data are transmitted over the data channel. Node with packets to transmit sends a RTS over the control channel and waits for CTS. If no CTS arrive then the node enters aback-off state. 
Node 3

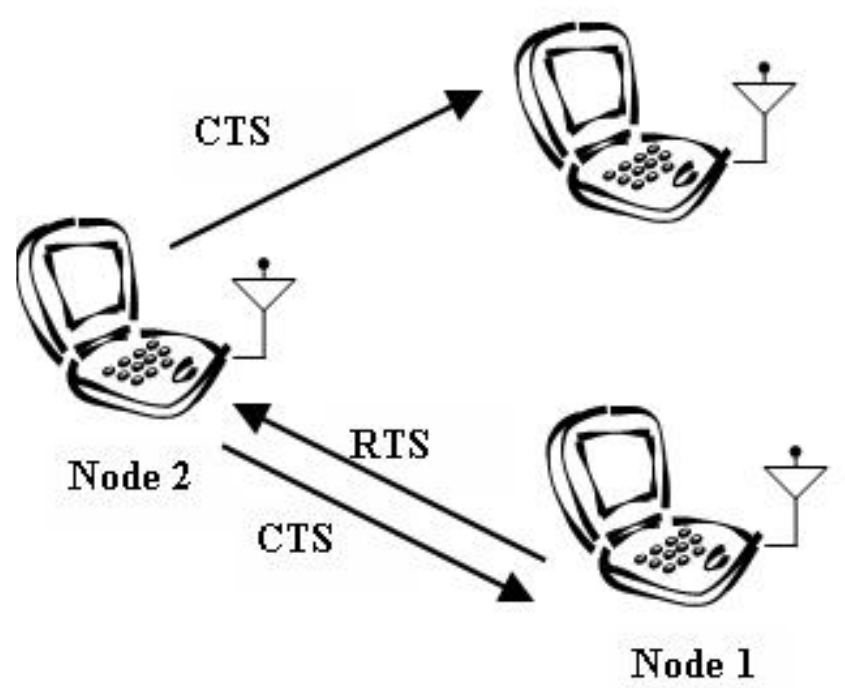

Figure 3.Flow Diagrams of RTS/CTS

However, if CTS is received then the node transmits the data packets over the data channel. The receiving node transmits a "busy tone" over the control channel for others to determine that the data channel is busy. The use of control channel allows nodes to determine when and how long to power off. If a node has no packets to transmit, that node ought to power itself off.

\subsection{Proposed Solution:}

Wireless medium is inherently broadcast in nature. When a node transmits data then multiple nodes (which are its neighbours) can receive the data. This paper aims at exploiting the broadcast nature of wireless medium and communication cost to reduce the energy consumption in the network. When a node detects an event, it knows whether any of its neighbours have already reported the same event or not. If reported then it refrains itself from reporting. So the number of packets sends to report the event reduces and hence energy consumption in sensor nodes is also reduced. When an event is detected by a sensor node, it waits for a time period before reporting the event to the sink. This wait time depends on the following factors

- The intensity of the event detected

- Residual energy of the node

- Communication cost to the nearest neighbour on the route to sink

If the node can overhears more than $\mathrm{N}$ nodes reporting the same event during this wait time period, it abandons the attempt to report the event. Given for each of the above factors depending on whether it is more interested in precision of data or in reducing the energy consumption. This is only a broad outline of the solution and details of implementation of this scheme are yet to be worked out.

We have considered the industrial wireless sensor environment which consists of multiple source nodes and single sink node. The model is design for multi-hop and one hop situation. The simulation area is considered as 2500 meters by 1000 meters. The all other parameters which are considered formulation areas had shown in table no 1 and table no2. 
Table 1.Simulation Parameters

\begin{tabular}{|c|c|}
\hline Channel Type & Wireless Channel \\
\hline Radio Propagation Model & Two Ray Ground \\
\hline Antenna Model & Omni Antenna \\
\hline Network Interface Type & Wireless Physical \\
\hline MAC Type & 802.11 \\
\hline Routing Type & APCRP \\
\hline Interface Queue Type & $\begin{array}{c}\text { PriQueue/CMU } \\
\text { PriQueue }\end{array}$ \\
\hline Buffer Size of IFq & 50 \\
\hline
\end{tabular}

Table 2.Node Configuration Parameters

\begin{tabular}{|l|l|}
\hline Simulation area & $250 \mathrm{~m}^{*} 100 \mathrm{~m}$ \\
\hline Model & Energy \\
\hline Initial Energy & $100 \mathrm{~J}$ \\
\hline Transmitting Power & $0.6 \mathrm{mw}$ \\
\hline Receiving Power & $0.9 \mathrm{mw}$ \\
\hline Transmission Range & $250 \mathrm{~m}$ \\
\hline
\end{tabular}

\section{Simulation Process}

The NS-2 software is used for the simulation.

\subsection{Animation Results:}

\subsection{Simulation Results:}

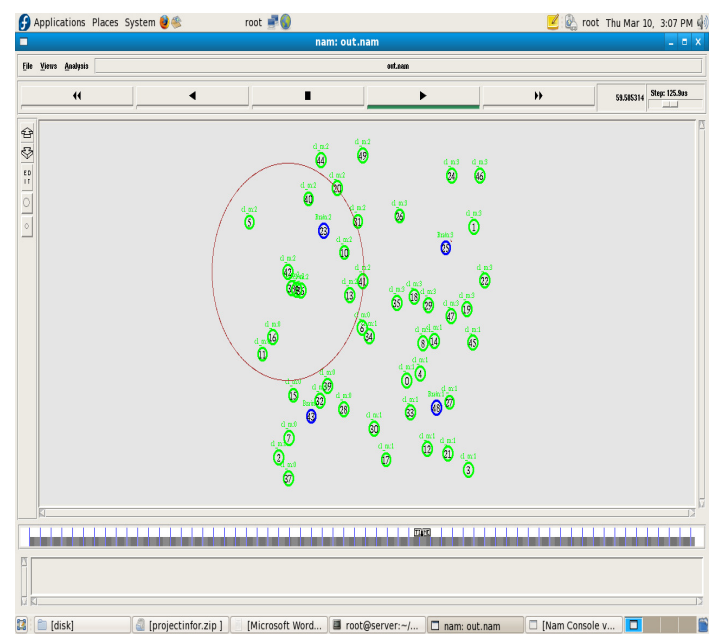

Figure 4.NAM output

\subsubsection{Throughput:}

The rate of data packets arrived to the gateway. 
International Journal of Distributed and Parallel Systems (IJDPS) Vol.2, No.5, September 2011

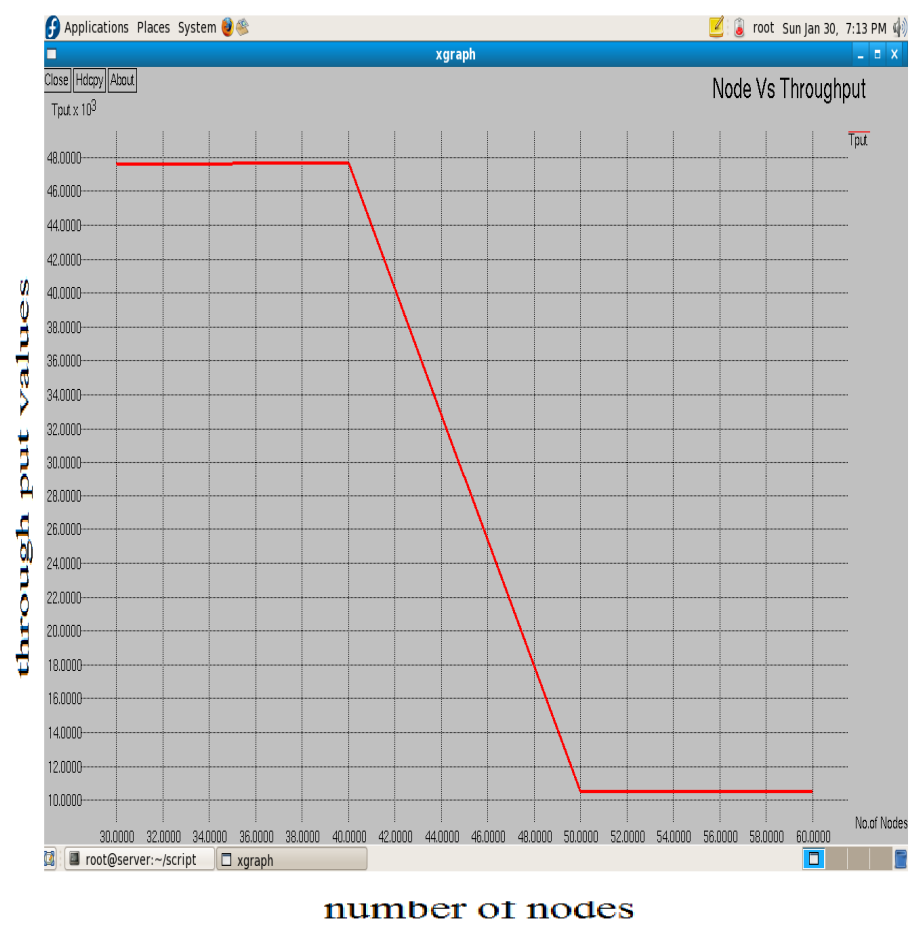

Figure 5.Node Vs Throughout

\subsubsection{End-to-End Delay:}

The time it takes a data packet from the sensing node to the gateway.

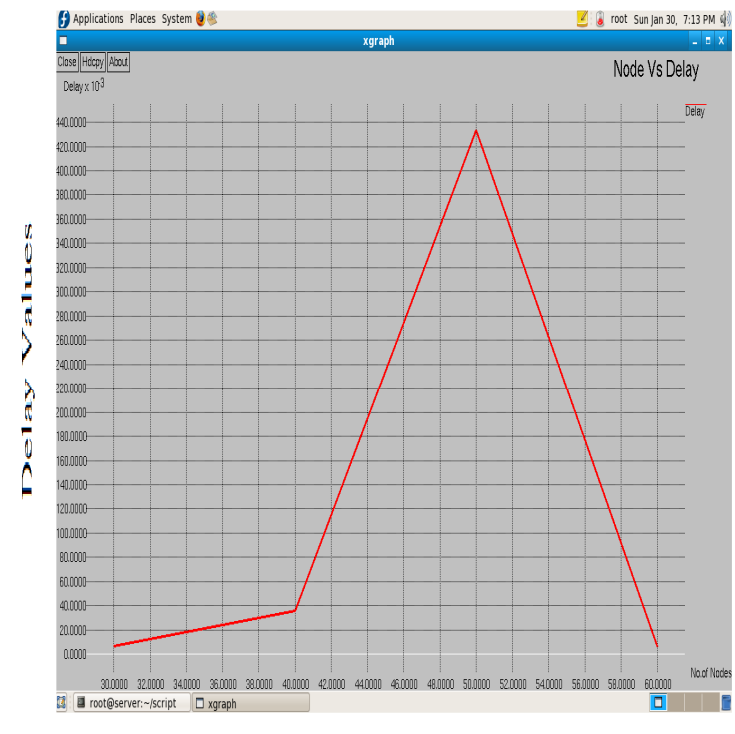

number of nodes

Figure 6.Node Vs Delay 
International Journal of Distributed and Parallel Systems (IJDPS) Vol.2, No.5, September 2011

\subsubsection{Average energy consumed per packet:}

The average energy consumed in transmitting and receiving a data packet.

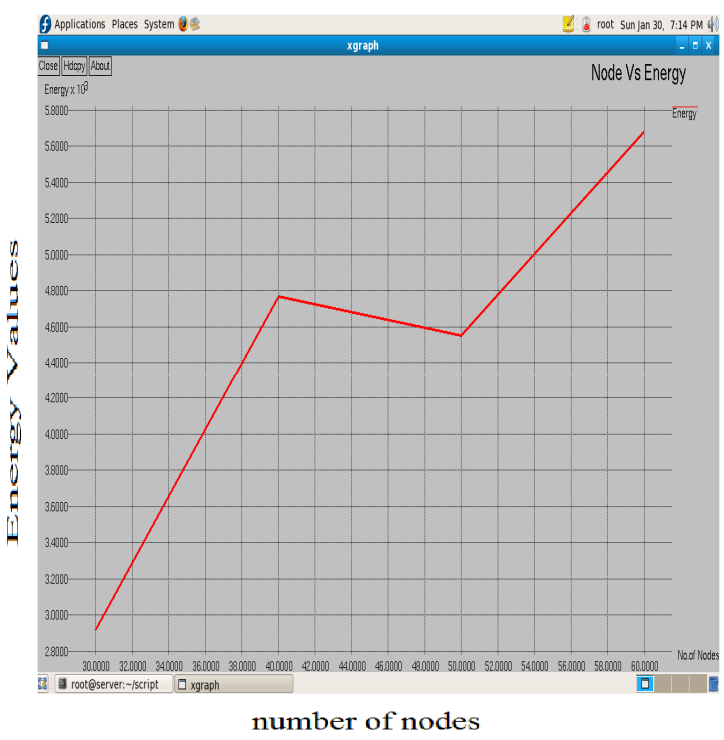

Figure 7.Node Vs Energy

\subsection{PDR (Packet Delivery Ratio) graph:}

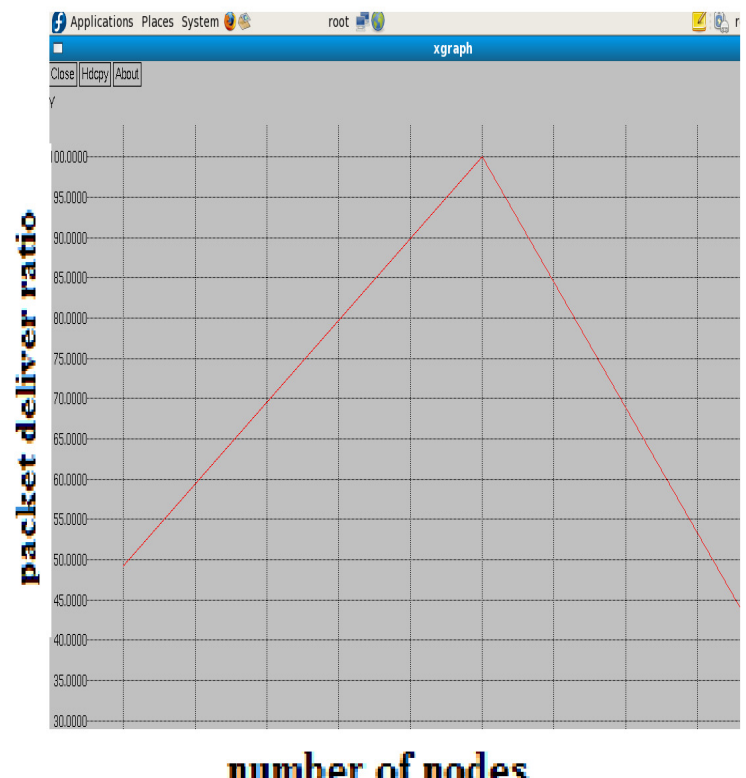

Figure 8.PDR Transmission 
International Journal of Distributed and Parallel Systems (IJDPS) Vol.2, No.5, September 2011

\section{Conclusion}

A new MAC protocol, Energy Aware Routing protocol (APCRP), based on TDMA technique is proposed to avoid the interference problem. The APCRP protocol significantly reduces the total number of route request packets, increase the packet delivery ratio, decreasing end- to-end delays for the data packets, lower control overhead, fewer collisions of packets, supporting reliability and decreasing the power consumption.

This paper is being simulated and solutions are realized in Ns-2(Network Simulator) to prove assumptions considered in current work. Ns-2 is an object-oriented event-driven simulator with extensive support for simulation of MAC protocol.

\section{References}

1. I. F. Akyildiz, W. Su, et al, "A survey on sensor networks", Communications Magazine, IEEE, vol. 40, no. 8, pp.102-114, 2002.

2. R. S. Chang, C. J. Kuo, "An Energy Efficient Routing Mechanism for Wireless Sensor Networks," Proceedings of the 20th International Conference on Advanced Information Networking and Applications, Vienna, Austria, pp.308-312, 2006.

3.W.Ye,J. Jeidemann,D. Estrin, An energy-efficient MAC protocol for wireless sensor networks,in:Proceedings of the IEEE INFOCOM ,2002.

4.. P. Chen, B. O'Dea, and E. Callaway, "Energy efficient system design with optimum transmission range for wireless ad hoc networks," in Proc. IEEE ICC, 2002, pp. 945-952.

5. J. L. Gao, "Analysis of energy consumption for ad hoc wireless sensor networks using a bit-meter-perjoule metric," IPN Progress Report 42-150, August 2002.

6.R. Madan and S. Lall, "Distributed algorithms for maximum lifetime routing in wireless sensor networks," IEEE Trans. Wireless Commun., vol. 5, no. 8, pp. 2185-2193, Aug. 2006.

7. Manish Kumar jha, Atul kumar pandey, Dipankar pal, "Energy-efficient multi-layer MAC (MLMAC) protocol for wireless sensor networks" Int.J.Electron.Commun.209-216.

8.Wireless Sensor Networks: An information processing approach-feng zhao, leonidas guibas, ISBN: 978-1-55860-914-3 @2004 by Elsevier Inc.

9. Tijs van Dam and Koen Langendeon "An Adaptive Energy Efficient MAC Protocol for Wireless Sensor Networks" , ACM-Proceedings of the $1^{\text {st }}$ international conference on Embedded networked sensor systems, 2003 .

10..ECCRA: An energy-efficient coverage and connectivity preserving routing algorithm under border effects in wireless sensor networks; Yan Jin , Ju-Yeon Jo , Ling Wang, Yoohwan Kim , Xiaozong Yang,Computer Communications 31 (2008) 2398-2407

11. An energy-efficient, transport,-controlled MAC protocol for wireless sensor networks; Jaesub Kim *, Kyu Ho Park, computer Networks53(2009) 1879-1902 .

12. W. Wang, H. Wang, D. Peng, H. Sharif, An energy efficient pre schedule scheme for hybrid CSMA/TDMA MAC in wireless sensor networks, in: Proceedings of 10th IEEE Singapore International Conference on Communication Systems, 2006, pp. 1-5.

13. J. van Greunen and J. Rabaey. Lightweight Time Synchronization for Sensor Networks. In Proc.2nd ACM Intl. Workshop on Wireless Sensor Networks and Applications (WSNA), San Diego, CA, September 2003.

14. An adaptive CSMA/TDMA hybrid MAC for energy and throughput improvement of wireless sensor networks Mohammad Hossein Sedighi Gilani, Iman Sarrafi, Maghsoud Abbaspour (2011), doi:10.1016/j.adhoc.2011.01.005. 
International Journal of Distributed and Parallel Systems (IJDPS) Vol.2, No.5, September 2011

S.Koteshwara rao graduated in Electronics and Communication Engineering from Dr.S.G.I.E.T and post graduated in instrumentation and Control Systems from JNTUK and pursuing his PhD from JNTUK and research interests includes MAC Layer issues of Wireless sensor networks and Digital communication.

Dr.M.Sailaja obtained B.E in Electronics \&Communication Engineering. She Completed M.S in USA and PhD from JNTU. Presently, she is working as HOD in ECE Department in JNTU Kakinada. Her Research interests include Parallel Processing and Computer Networks.

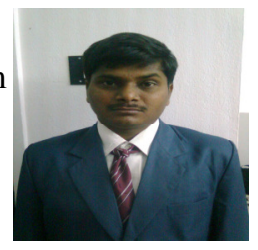

Dr. Tenneti Madhu obtained his B.E.degree from University of Madras, M.Tech from REC, Kurukshetra in 1994 and Ph.D from Osmania University in 2004. His research interests include GPS Data Analysis, Image Processing, Nano Technology and VLSI design.

P. Ramesh graduated in Electronics \& Communication Engineering from RMCE, Hyderabad (India) and post graduated in Embedded systems from VNRVJIET, Hyderabad. His research interests includes Cluster based routing for Wireless Sensor Networks and Digital Communication.
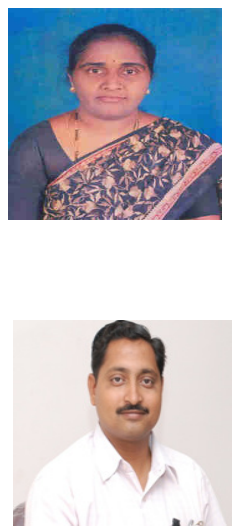

V.Rajesh graduated in Electronics Engineering from the Institution of Engineers (India)and post graduated in Instrumentation from SRTM and currently submitted his PhD Thesis from ECE dept, Andhra University and research interests includes measuring and processing of BioElectric Signals, Virtual Instrumentation and Image processing.
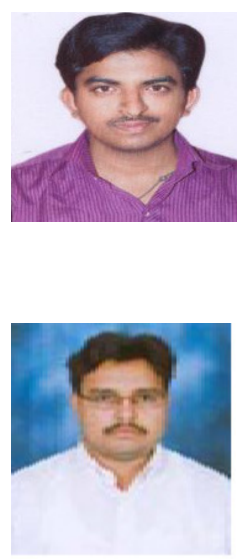